\title{
Alerta bibliográfica: revisiones sistemáticas de parálisis cerebral
}

La Subdirección de Investigación de Teletón Chile, genera una alerta bibliográfica de las revisiones de parálisis cerebral publicadas en PubMed.

Publicamos en este número de la revista Rehabilitación Integral un listado de los títulos aparecidos entre junio y noviembre de 2019 en inglés y traducidos al español, y algunos con resumen en español.

Las revisiones del tema parálisis cerebral indexadas en PubMed Clinical Queries incluyen las citas sobre las revisiones sistemáticas, metaanálisis, revisiones de ensayos clínicos, medicina basada en la evidencia, conferencias de consenso y guías clínicas. Filtro en PubMed: cerebral palsy AND systematic [sb].

El servicio de alerta contiene las citas indicando:

Cita completa

Título traducido al español

Resumen en español para algunos artículos.

Para artículos publicados en línea previo a ser editados se agrega uno o más de los identificadores:

PMID: PubMed Unique Identifier (Identificador único PubMed).

doi: Digital Object Identifier (Identificador de objeto digital).

\section{Índice de junio de 2019}

1. Efectos de la estimulación de corriente continua transcraneal (tDCS) en la mejora del equilibrio: revisión sistemática $y$ metaanálisis.

Effects of transcranial direct current stimulation (tDCS) on balance improvement: a systematic review and meta-analysis.

de Moura MCDS, Hazime FA, Marotti Aparicio LV, Grecco LAC, Brunoni AR, Hasue RH. Somatosens Mot Res. 2019 Jun 11: 1-14.

2. Tratamiento del desarrollo neurológico (Bobath) para niños con parálisis cerebral: revisión sistemática.

Neurodevelopmental Treatment (Bobath) for Children With Cerebral Palsy: A Systematic Review.
Zanon MA, Pacheco RL, Latorraca COC, Martimbianco ALC, Pachito DV, Riera $R$. J Child Neurol. 2019 Jun 10:883073819852237. doi: 10.1177/0883073819852237. [Epub ahead of print].

\section{Anomalías congénitas en niños con pará- lisis cerebral: revisión sistemática. \\ Congenital Anomalies in Children With Ce- rebral Palsy: A Systematic Review. \\ Goldsmith S, McIntyre S, Hansen M, Badawi N. J Child Neurol. 2019 Jun 17:883073819854595. doi: 10.1177/0883073819854595. [Epub ahead of print]}

\section{Calidad de vida en niños y adolescentes con parálisis cerebral: revisión sistemática con metaanálisis. \\ Quality of life in children and adolescents with cerebral palsy: a systematic review with meta-analysis. \\ Makris T, Dorstyn D, Crettenden A. \\ Disabil Rehabil. 2019 Jun 10: 1-10.}

\section{Resumen}

Antecedentes: La parálisis cerebral se ha relacionado con la disminución de la calidad de vida. Sin embargo, el uso de las perspectivas auto-versus proxy y las comparaciones de la muestra con la norma para examinar el impacto en la calidad de vida puede producir resultados diferentes. Objetivo: Comparar las clasificaciones de calidad de vida en niños y adolescentes con parálisis cerebral en comparación con los compañeros con desarrollo típico en consideración de la muestra y moderadores metodológicos sobre los efectos estimados. Métodos: Se identificaron once estudios independientes, que incluían una muestra combinada de 1.475 familias que vivían con parálisis cerebral y 42.119 compañeros. La calidad de los informes del estudio se evaluó con la herramienta QualSyst y se calcularon las diferencias medias estandarizadas (Hedges ' $\mathrm{g}$ ) con los intervalos de confianza asociados y los valores de p. La heterogeneidad se examinó utilizando un modelo de efectos aleatorios. Resultados: Todos los estudios proporcionaron de buena a excelente detalle metodológico y 
estadístico. La calidad de vida física se deterioró significativamente entre las personas con parálisis cerebral (rango de g: -0,42 a -1,58). Sin embargo, se observaron hallazgos inconsistentes en relación con el efecto de la parálisis cerebral sobre la calidad de vida social y psicológica (rango g: 0,04 a -0,80) (rango g: -0,80 a -0,51), según la medida utilizada. Hubo una tendencia a que los padres evaluaran la calidad de vida física de sus hijos por debajo de los puntajes informados por los niños. Conclusiones: La calidad de vida física es, invariablemente, más afectada en las personas con parálisis cerebral. La conexión entre parálisis cerebral y calidad de vida psicosocial es menos clara. Las notables discrepancias entre padres e hijos resaltan el valor de un enfoque multiinformativo para la evaluación de la calidad de vida de los niños. Implicaciones para la rehabilitación: La calidad de vida es un importante resultado relacionado con la salud en la investigación y la práctica de la parálisis cerebral. La recopilación de datos de autoinforme y de representación puede ayudar a resaltar los problemas de calidad de vida que son relevantes para el padre y el niño o adolescente con parálisis cerebral. La selección del instrumento de calidad de vida adecuado depende del propósito de la evaluación, con las medidas disponibles que varían en su enfoque en la funcionalidad, la subjetividad y los elementos específicos de la enfermedad. La evaluación de la calidad de vida de los niños con parálisis cerebral debe extenderse más allá de las capacidades funcionales para incluir problemas menos obvios, pero críticos, psicológicos y sociales.

\section{Índice de julio de 2019}

\section{Deficiencia en la alimentación y la deglu- ción en niños con accidente cerebrovascular y parálisis cerebral unilateral: revisión sistemática.}

Feeding and swallowing impairment in children with stroke and unilateral cerebral palsy: a systematic review.

Sherman V, Greco E, Moharir M, Beal D, Thorpe K, Martino R.

Dev Med Child Neurol. 2019; 61 (7): 761-9.

\section{Resumen}

Objetivo: Esta revisión sistemática se centró en las estimaciones de frecuencia de disfagia (problemas de alimentación y deglución), resultados de salud relacionados y carga del cuidador en niños con accidente cerebrovascular o parálisis cerebral unilateral (PC). Método: Se realizaron búsquedas en seis bases de datos electrónicas desde su inicio hasta noviembre de 2017, junto con una búsqueda manual de ocho revistas relevantes. Dos evaluadores evaluaron los resúmenes y los artículos completos para determinar su elegibilidad. Las discrepancias se resolvieron por consenso. Los artículos aceptados fueron evaluados por su calidad. Los datos fueron extraídos y analizados descriptivamente. Resultados: De los 1.660 resúmenes, cinco cumplieron con los criterios de inclusión, de los cuales tres se centraron en el accidente cerebrovascular y dos PC unilaterales. Entre los estudios, las definiciones operativas de alimentación y deglución variaban. No se proporcionaron detalles suficientes sobre los métodos de evaluación y el calendario. Las frecuencias reportadas de disfagia variaron de $24,2 \%$ a $88,6 \%$. Un estudio informó los resultados de salud relacionados con la disfagia y ninguno informó la carga del cuidador. Interpretación: Estos resultados sugieren que la disfagia es común en niños con accidente cerebrovascular y PC unilateral; sin embargo, aún se desconoce su frecuencia y su impacto en la salud y la carga del cuidador. La disponibilidad de una herramienta estandarizada para identificar con precisión la disfagia en estos niños es un primer paso recomendado para abordar esta brecha en la evidencia. Aporte de este paper: Hay datos limitados sobre la incidencia de disfagia después de un accidente cerebrovascular infantil y parálisis cerebral unilateral. La evidencia disponible muestra frecuencias de disfagia reportadas de $24,2 \%$ a $88,6 \%$. Sólo un estudio informó resultados de salud relacionados con disfagia. Ningún estudio informó sobre la carga del cuidador.

2. Fórmula enriquecida con nutrientes versus fórmula estándar para bebés prematuros. Nutrient-enriched formula versus standard formula for preterm infants. 
Walsh V, Brown JVE, Askie LM, Embleton ND, McGuire $W$.

Cochrane Database Syst Rev. 2019 Jul 17;7:CD004204.

doi: 10.1002/14651858.CD004204.pub3. [Epub ahead of print].

3. Cuantificación de la atrofia de la materia gris profunda utilizando enfoques de segmentación automatizados: revisión sistemática de estudios de resonancia magnética estructural.

Quantifying deep grey matter atrophy using automated segmentation approaches: A systematic review of structural MRI studies. Pagnozzi AM, Fripp J, Rose SE.

Neuroimage. 2019 Jul 15; 201: 116018. doi: 10.1016/j.neuroimage.2019.116018. [Epub ahead of print].

4. Tratamiento de fisioterapia en niños con parálisis cerebral después de una cirugía multinivel de evento único: revisión sistemática cualitativa. Un primer paso hacia una guía clínica para la fisioterapia después de una cirugía multinivel de evento único. Physical therapy treatment in children with cerebral palsy after single-event multilevel surgery: a qualitative systematic review. A first step towards a clinical guideline for physical therapy after single-event multilevel surgery. van Bommel EEH, Arts MME, Jongerius PH, Ratter J, Rameckers EAA.

Ther Adv Chronic Dis. 2019; 10: 2040622319 854241.

doi: $10.1177 / 2040622319854241$.

5. Efectividad del juego interactivo en computadora sobre el equilibrio y el control postural para niños con parálisis cerebral: revisión sistemática.

Effectiveness of interactive computer play on balance and postural control for children with cerebral palsy: A systematic review.

Pin $T W$.

Gait Posture. 2019; 73: 126-39.

6. Intervenciones para el tratamiento de enfermedad respiratoria en jóvenes con parálisis cerebral: revisión sistemática.
Interventions for management of respiratory disease in young people with cerebral palsy: A systematic review.

Blackmore AM, Gibson N, Cooper MS, Langdon K, Moshovis L, Wilson AC.

Child Care Health Dev. 2019 Jul 5.

doi:10.1111/cch.12703. [Epub ahead of print].

7. Efectos de la cirugía de las extremidades superiores sobre las actividades y la participación de niños con parálisis cerebral: revisión sistemática.

Effects of upper extremity surgery on activities and participation of children with cerebral palsy: a systematic review.

Louwers A, Warnink-Kavelaars J, Daams J, Beelen A.

Dev Med Child Neurol. 2019 Jul 23.

doi: $10.1111 / \mathrm{dmcn} .14315$. [Epub ahead of print].

8. Prevalencia de problemas de babeo, deglución y alimentación en la parálisis cerebral a lo largo de la vida: revisión sistemática y metaanálisis.

Prevalence of drooling, swallowing, and feeding problems in cerebral palsy across the lifespan: a systematic review and meta-analyses.

Speyer R, Cordier R, Kim JH, Cocks N, Michou E, Wilkes-Gillan $S$.

Dev Med Child Neurol. 2019 Jul 22.

doi: $10.1111 / \mathrm{dmcn} .14316$. [Epub ahead of print]

\section{Índice de agosto de 2019}

1. Prevalencia de depresión, ansiedad y trastornos relacionados con sustancias en padres de niños con parálisis cerebral: revisión sistemática.

Prevalence of depression, anxiety, and substance-related disorders in parents of children with cerebral palsy: a systematic review.

Barreto TM, Bento MN, Barreto TM, Jagersbacher JG, Jones NS, Lucena R, Bandeira ID. Dev Med Child Neurol. 2019 Aug 5.

doi: $10.1111 /$ dmcn.14321. [Epub ahead of print].

\section{Resumen}

Objetivo: Estimar la prevalencia de enfermedades mentales en padres de niños con 
parálisis cerebral (PC). Método: Esta es una revisión sistemática que sigue los ítems de informes preferidos para los protocolos de revisión sistemática y metaanálisis en la búsqueda de estudios observacionales que determinen la prevalencia de depresión, ansiedad y abuso de sustancias en padres de personas con PC. Las fuentes de información utilizadas para este estudio fueron: PubMed, SciELO, Cochrane Library, Clinical Trials y Biblioteca Virtual de Saúde. Resultados: Se seleccionaron e incluyeron catorce artículos que investigaban a 1.264 madres y 105 padres de niños con PC. Los datos extraídos para el análisis se dividieron en tres categorías: datos del estudio, datos sobre los participantes con PC y datos sobre los padres. Todos los estudios incluyeron padres voluntarios, de los cuales el 95 por ciento eran mujeres. Interpretación: La PC está relacionada con una mayor prevalencia de síntomas de depresión y ansiedad en los padres. Factores como el grado de funcionalidad y el nivel socioeconómico de un niño pueden influir en la frecuencia de los trastornos mentales en los padres. Sin embargo, estos estudios tienen muestras heterogéneas y aplican diferentes criterios para caracterizar sus poblaciones. Aporte de este artículo: La depresión y la ansiedad son más frecuentes en los padres de niños con parálisis cerebral (PC) que en los padres de niños con desarrollo típico. La gravedad de la enfermedad del niño es un factor de riesgo de enfermedad mental en los padres de niños con PC. Cuanto más tiempo se dedica al cuidado infantil, mayor es el riesgo de enfermedad mental entre las madres de niños con PC. Hay una falta de datos confiables en la literatura sobre abuso de sustancias en padres de niños con PC.

\section{Revisión sistemática de las medidas de} actividad de las extremidades superiores para niños de 5 a 18 años con parálisis cerebral bilateral.

A systematic review of upper limb activity measures for 5- to 18-year-old children with bilateral cerebral palsy.

Burgess A, Boyd RN, Ziviani J, Sakzewski L. Aust Occup Ther J. 2019 Aug 5.

doi: 10.1111/1440-1630.12600. [Epub ahead of print].

\section{Índice de septiembre de 2019}

1. Entrenamiento de actividades en suelo en niños con parálisis cerebral: Revisión sistemática y metaanálisis.

Activity training on the ground in children with cerebral palsy: Systematic review and meta-analysis.

Bania T, Chiu HC, Billis E.

Physiother Theory Pract. 2019; 35 (9): 810-821.

2. El impacto de las intervenciones vocacionales en los resultados vocacionales, la calidad de vida y la integración comunitaria en adultos con discapacidades de inicio en la infancia: revisión sistemática.

The Impact of Vocational Interventions on Vocational Outcomes, Quality of Life, and Community Integration in Adults with Childhood Onset Disabilities: A Systematic Review. Ma Z, Dhir P, Perrier L, Bayley M, Munce $S$. J Occup Rehabil. 2019 Sep 18.

doi: 10.1007/s10926-019-09854-1. [Epub ahead of print].

\section{Resumen}

Propósito: A pesar del deseo y la capacidad de trabajar, las personas con discapacidades de inicio en la infancia están subrepresentadas en el empleo. Las intervenciones vocacionales alivian algunas barreras para obtener y mantener empleo para esta población. La pregunta de investigación abordada es: ¿Cuál es el impacto de las intervenciones vocacionales en los resultados vocacionales, la calidad de vida y la integración comunitaria en adultos con discapacidades neurológicas de inicio en la infancia, incluida la parálisis cerebral (PC), la espina bífida y la lesión cerebral adquirida (ABI)? Métodos: Se realizó una búsqueda bibliográfica en múltiples bases de datos electrónicas. Se incluyeron todos los estudios experimentales y observacionales con grupo(s) de comparación. Dos revisores completaron de forma independiente la selección de títulos y resúmenes, la evaluación de texto completo, la extracción de datos y la evaluación del riesgo de sesgo. Resultados: Diecisiete estudios fueron elegibles para la inclusión final, incluidos tres ensayos controlados aleatorios, cuatro estudios 
no aleatorios y diez estudios observacionales. Dieciséis de diecisiete estudios incluyeron solo individuos con $\mathrm{ABI}$, mientras que un estudio incluyó individuos con PC. Las intervenciones vocacionales realizadas en los estudios experimentales fueron principalmente componentes de intervenciones multifacéticas. La mayoría de los estudios observacionales fueron del Servicio de Rehabilitación Vocacional de los Estados Unidos. Conclusiones: Las intervenciones vocacionales pueden ser efectivas para mejorar los resultados vocacionales, la calidad de vida y la integración comunitaria para las personas con ABI. Hay evidencia experimental limitada sobre intervenciones que se dirigen específicamente al empleo. Los datos de observación sugieren que recibir asistencia para la colocación laboral, capacitación y apoyo en el trabajo, asesoramiento/orientación, mantenimiento y empleo con apoyo predijo con éxito los resultados del empleo.

3. Definiendo de terapia funcional en la investigación de niños con parálisis cerebral: revisión sistemática.

Defining Functional Therapy in Research Involving Children with Cerebral Palsy: A Systematic Review.

Geijen M, Ketelaar M, Sakzewski L, Palisano $R$, Rameckers $E$.

Phys Occup Ther Pediatr. 2019 Sep 26:1-16.

\section{Hipoterapia: revisión sistemática de ma- peo de investigaciones revisadas por pares, 1980 a 2018. \\ Hippotherapy: a systematic mapping review of peer-reviewed research, 1980 to 2018. \\ Wood WH, Fields BE. \\ Disabil Rehabil. 2019; 1-25.}

\section{Índice de octubre de 2019}

1. Efecto de las intervenciones de fisioterapia sobre los parámetros témporo-espaciales de la marcha en niños con parálisis cerebral: revisión sistemática.

Effect of physical therapy interventions on spatiotemporal gait parameters in children with cerebral palsy: a systematic review.

Corsi C, Santos MM, Moreira RFC, Dos Santos
AN, de Campos AC, Galli M, Rocha NACF. Disabil Rehabil. 2019 Oct 7: 1-10. doi: 10.1080/09638288.2019.1671500. [Epub ahead of print].

2. Revisión sistemática de estudios clínicos controlados que utilizan sangre del cordón umbilical para terapia regenerativa: identificación de barreras para evaluar la eficacia. Systematic review of controlled clinical studies using umbilical cord blood for regenerative therapy: Identifying barriers to assessing efficacy.

Aziz J, Liao G, Adams Z, Rizk M, Shorr R, Allan DS.

Cytotherapy. 2019 Oct 3.

doi: 10.1016/j.jcyt.2019.08.004. [Epub ahead of print] Review.

3. Impacto de la terapia resistida sobre los parámetros de la marcha en niños con parálisis cerebral: revisión sistemática y metaanálisis.

[Impact of resistive therapy on gait parameters in children with cerebral palsy: systematic review and meta-analysis].

Collado-Garrido L, Paras-Bravo P, CalvoMartin P, Santibáñez M.

Rev Neurol. 2019; 69 (8): 307-316.

4. Revisión sistemática de las deformidades espinales después de la rizotomía dorsal selectiva multinivel.

Systematic review of spinal deformities following multi-level selective dorsal rhizotomy. Wheelwright M, Selvey PJ, Steinbok P, Singhal A, Ibrahim $G$, Fallah A, Weil AG, Halvorson K, Tu $A$.

Childs Nerv Syst. 2019 Oct 8.

doi: 10.1007/s00381-019-04375-x. [Epub ahead of print].

5. La toxina botulínica tipo A en el tratamiento de la espasticidad de las extremidades inferiores en niños con parálisis cerebral. Botulinum toxin type $\mathrm{A}$ in the treatment of lower limb spasticity in children with cerebral palsy. Blumetti FC, Belloti JC, Tamaoki MJ, Pinto JA. Cochrane Database Syst Rev. 2019 Oct 8; 10: CD001408. 
doi: 10.1002/14651858.CD001408.pub2. [Epub ahead of print] Review.

\section{La fiabilidad y validez de la prueba Timed up and Go como una herramienta clínica en personas con y sin discapacidad a través de un ciclo de vida: revisión sistemática}

The reliability and validity of the Timed Up and Go as a clinical tool in individuals with and without disabilities across a lifespan: a systematic review.

Christopher A, Kraft E, Olenick H, Kiesling $R$, Doty $A$.

Disabil Rehabil. 2019 Oct 26: 1-15. doi: 10.1080/09638288.2019.1682066. [Epub ahead of print].

7. Efecto de la terapia de ondas de choque extracorpóreas sobre la espasticidad muscular en pacientes con parálisis cerebral: metaanálisis y revisión sistemática.

Effect of extracorporeal shock wave therapy on muscle spasticity in patients with cerebral palsy: meta-analysis and systematic review. Kim HJ, Park JW, Nam K.

Eur J Phys Rehabil Med. 2019 Oct 15. doi: 10.23736/S1973-9087.19.05888-X. [Epub ahead of print].

\section{Resumen}

Introducción: Recientemente, se han realizado ensayos clínicos para evaluar la eficacia de la terapia de ondas de choque extracorpóreas (ESWT) en pacientes con parálisis cerebral (PC). Sin embargo, varios estudios adoptaron diferentes escalas clínicas, por lo que no es posible llegar a una conclusión definitiva sobre la eficacia de ESWT para reducir la espasticidad en PC. El objetivo de este metaanálisis fue evaluar los efectos de ESWT en la reducción de la espasticidad después de aplicar ESWT en pacientes con PC. Adquisición de pruebas: De acuerdo con la declaración de PRISMA, los autores realizaron búsquedas en MEDLINE, EMBASE, Web of Science, Cochrane Central Register of Controlled Trials y Scopus desde sus fechas de inicio hasta el 11 de diciembre de 2018. Se incluyeron ensayos controlados aleatorios en cualquier idioma que usaran ESWT con el fin de mejorar espasticidad en pacientes con PC. Evaluamos la espasticidad medida por la Escala de Ashworth modificada (MAS), el rango de movimiento (ROM) y los valores baropodométricos como resultados. Síntesis de evidencia: Dos autores extrajeron y verificaron datos en forma independiente.Se realizó metaanálisis donde fue posible, y por lo demás, se sintetizó datos en forma narrativa. De un total de 206 artículos, seleccionaron 16 y 5 de ellos se incluyeron en este metaanálisis. Conclusiones: El grado de MAS como resultado primario mejoró significativamente después de ESWT en comparación con el del grupo control (diferencia de medias [DM]: -0,62; intervalo de confianza [IC] de 95\%: - 1,52 a -0,18). La ROM después de ESWT también mejoró significativamente en comparación con la de los grupos de control (DM: 18,01; IC 95\%: 6,11 a 29,91). Las medidas baropodométricas mostraron aumentos significativos en el área de contacto del pie durante la marcha (DME: 29,00; IC de 95\%: 11,08 a 46,92), pero no significativamente en la presión máxima bajo el talón (DM: 15,12; IC de 95\%: -1,85 a 32,10$)$ inmediatamente después ESWT. No se observaron efectos secundarios graves en ningún paciente después de ESWT. ESWT puede ser una alternativa válida a las opciones de tratamiento existentes que apuntan a la disminución de la espasticidad y la mejora del ROM en pacientes con PC para mantener estilos de vida saludables y normalizar el patrón de marcha espástica. Es necesario establecer una mayor estandarización de los protocolos de tratamiento, incluidos los intervalos e intensidades de tratamiento, y se necesitan estudios de seguimiento a largo plazo para verificar nuestros resultados.

\section{Índice de noviembre de 2019}

1. Manejo de la espasticidad muscular en ninos con parálisis cerebral mediante la terapia de ondas de choque extracorpóreas: revisión sistemática.

Management of muscle spasticity in children with cerebral palsy by means of extracorporeal shockwave therapy: a systematic review of the literature. 
Corrado B, Di Luise C, Servodio Iammarrone C. Dev Neurorehabil. 2019 Nov 1:1-7.

2. Revisión sistemática y metaanálisis de la prevalencia de problemas del sueño en niños

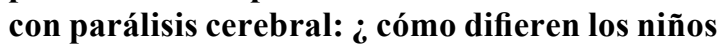
con parálisis cerebral entre sí y de los niños con desarrollo típico?

A systematic review and meta-analysis of the prevalence of sleep problems in children with cerebral palsy: how do children with cerebral palsy differ from each other and from typically developing children?

Horwood L, Li P, Mok E, Shevell M, Constantin E. Sleep Health. 2019 Dec;5(6):555-571. doi: 10.1016/j. sleh.2019.08.006. Epub 2019 Nov 15.

3. Efecto de la terapia de realidad virtual sobre el equilibrio y la marcha en niños con parálisis cerebral: Revisión sistemática.

Effect of Virtual Reality Therapy on Balance and Walking in Children with Cerebral Palsy: A Systematic Review.

Warnier N, Lambregts S, Port IV.
Dev Neurorehabil. 2019 Nov 1:1-17. doi: 10.1080 /17518423.2019.1683907. [Epub ahead of print].

4. Revisión sistemática sobre evaluación de calidad de vida en adultos con parálisis cerebral: problemas desafiantes y un llamado a la investigación.

A systematic review on quality of life assessment in adults with cerebral palsy: Challenging issues and a call for research.

Alves-Nogueira AC, Silva N, McConachie H, Carona $C$.

Res Dev Disabil. 2019 Nov 6;96:103514. doi: 10.1016/j.ridd.2019.103514. [Epub ahead of print].

5. La relación entre necesidades especiales y trauma dental. Revisión sistemática y metaanálisis. The relationship between special needs and dental trauma. A systematic review and meta-analysis. Silveira ALNDMES, Magno MB, Soares TRC.

Dent Traumatol. 2019; 00: 1-19. https://doi. org/10.1111/edt.12527. 